\title{
The mysterious virus called "isn't"
}

\author{
The first close scrutiny of reports of a new virus supposed to cause symptoms of AIDS in people with neither HIV- \\ 1 nor HIV-2 suggests that there is no new virus.
}

CAN one have AIDS without the AIDS virus? Or is it possible that a third AIDS virus (neither HIV-1, the commonest form of the virus in the United States and Europe, nor HIV-2, which appears to be endemic in Africa) is lurking somewhere in the tissues of immunosuppressed individuals who have precariously low concentrations of CD4 T cells - the lymphocytes whose progressive depletion is associated with the development of AIDS?

Worldwide concern over the possibility that a previously unidentified infectious AIDS-like virus may be causing severe immunodeficiency disease was ignited last month by an article in Newsweek that asked, "Is a New AIDS Virus Emerging? The patients are sick or dying and most have risk factors. What they don't have is HIV."

Last week, researchers from the US National Institutes of Health (NIH) and Centers for Disease Control (CDC), together with AIDS and infectious disease experts from institutions all over the United States, held a one-day conference at CDC in Atlanta to assess data from several laboratories reporting retroviral activity in the cells of patients who appear not to be infected by HIV.

They reached a consensus on several points.

First, as Anthony S. Fauci, AIDS coordinator for NIH put it, "It is essential that we keep an open mind."

Second, present data do not support the suggestion that there is a third virus, which is why some are calling it the "isn't" virus. (The mystery virus has also been dubbed "MTV" for media transforming virus in the light of all the press attention it has received.)

Third, there is even less evidence that the alleged virus or virus particles, were they to be confirmed, cause disease.

Fourth (and most important), members of two expert panels - one to evaluate epidemiological data, the other retrovirology find nothing to suggest they are dealing with a new transmissible agent. This point is central to the issue underlying current anxiety - namely, the safety of the blood supply. The analogy has been made to the early days of the AIDS epidemic when scientists were uncertain what they were dealing with, but the analogy does not hold. From the outset, AIDS cases appeared in clusters, particularly in California and New York, and there was evidence that physicians were dealing with an infectious disease. But that is not the case here.

The Newsweek article, faxed to reporters in Amsterdam where the international AIDS meeting was in full swing during the week of 20 July, based its arresting question on the work of a couple of research teams who have reported CD4 patients. One team is that of Jeffrey Laurence of Cornell University Medical College in New York, who reported in the 1 August issue of The Lancet $(340,273 ; 1992)$ on five patients (four of them at risk for AIDS) who have low counts of CD4 T cells, various opportunistic infections and no apparent signs of HIV-1 or HIV-2 in the blood. In the Lancet article, not published until after Newsweek hit the faxes, Laurence suggests that the cases he describes "raise the question of the existence of other agents associated with transmissible immune deficiencies that can evade current laboratory detection techniques".

One of Laurence's co-authors, Stephen Morse of Rockefeller University, acknowledges that they may be seeing an artefact, a contaminant or viral activity from an endogenous retrovirus. But, he says, the issue is still "open" and research will proceed. "Our working hypothesis is still to look for a virus." Data similar to those from Laurence's laboratory were presented in Atlanta by David Ho of the Aaron Diamond AIDS Center in New York. He has seen more than a dozen patients, several of whom do not have the usual risk factors associated with AIDS - drug use, a history of blood transfusions or multiple homosexual partners. Two of them have signs of reverse transcriptase activity, but none is seropositive for HIV. Ho sent his material to an outside laboratory expert in retrovirus detection, but the answer came back negative.

The data that have probably received the lion's share of press attention were reported by Sudhir Gupta and colleagues at the University of California at Irvine. In a paper in the 15 August issue of the Proceedings of the NationalAcademy of Sciences $(\mathbf{8 9}, 7831$ $7835 ; 1992)$, which was released early in response to the public furore in Amsterdam, Gupta reports detection of a human intracisternal retroviral particle associated with CD4 T-cell deficiency in a 66-year-old woman with $P$. carinii pneumonia and her healthy, but CD4-depleted, daughter. (Gupta and the university have applied for a patent on the particle.)

Gupta's data are viewed with considerable scepticism by retrovirologists, several of whom have summed up their evaluations by saying, "there is nothing significant there".

Retroviral particles (particularly those known as A-type) have been studied on and off for years, in animal and human systems, without evidence that they cause disease. In his paper Gupta reports that the particle he found (and which he calls both a particle and a virus) is distinct from A-type particles. His particles do have gag and pol genes as HIV does, but lack the envelope gene that would allow the particles to get out of a cell. So the probability that they are infectious is said to be close to nil.

A fourth report at the Atlanta meeting by Robert F. Garry of Tulane University School of Medicine recapitulated two-year old data on retroviral particles and Sjögren's syndrome - an autoimmune disease that causes dryness of the eyes and mouth. In a paper in Science $(250,1127-1129 ; 1990)$ Garry and his colleagues reported a human intracisternal A-type retroviral particle in Sjögren's patients that is antigenically related to HIV but is distinguishable from it by ultrastructural and enzymatic analysis. Garry concludes not that the A-type particle causes Sjögren's syndrome but that the data support "an association between retrovirus infections and autoimmune phenomena that has long been suspected." An association, as yet unexplained etiologically or mechanistically (and probably unrelated to AIDS), may be what is emerging from the current reports of CD4-depleted patients.

So, where do things now stand? According to Fauci, there is general agreement that together Ho, Laurence, Gupta and others who have CD4-deficient patients may have identified a new and heterogeneous syndrome. "It could be decades old", he says. "It could just be coming to light because, for the past five years or so, we've been testing for CD4. We just don't know yet."CDC is calling the new syndrome ICL for idiopathic CD4 lymphopenia and there seems to be a consensus that it is epidemiologically and clinically real. The virology is more of a mystery but, Fauci says, the scepticism that greeted early reports in Amsterdam of a new virus "has really been reinforced by our analysis so far".

But good science and good public policy demand that the questions be more clearly resolved. NIH and CDC have established disease registries for CD4-deficient patients whose immune deficiency cannot be explained.

NIH and CDC have also established a repository for blood and other tissues from CD4-depleted patients that can be distributed to laboratories around the world for studies that may help to resolve this intriguing mystery.

Barbara J. Culliton 